\title{
La incorporación de la perspectiva de género en el Plan General Estructural de Castelló: objetivos, método, acciones y hallazgos
}

\author{
Gender mainstreaming in Castelló General Structural Planning: \\ goals, method, actions and findings
}

\author{
Eva M. Álvarez Isidro ${ }^{1}$ y Carlos J. Gómez Alfonso 3 \\ Fecha de recepción: 30-03-2018 - Fecha de aceptación: 23-07-2018 \\ Hábitat y Sociedad (ISSN 2173-125X), n. ${ }^{\circ} 11$, noviembre de 2018, pp. 201-219. \\ http://dx.doi.org/10.12795/HabitatySociedad.2018.i11.12
}

\begin{abstract}
In the work addressed for the incorporation of the gender perspective in Castelló (Valencian Community, Spain), the circumstance of an exceptional municipal technical team, led by Consuelo Leal, and a political government team that respects the decisions and technical recommendations have coincided. The advisory team on gender issues - redactor of this text- has contributed, fundamentally, to the implementation of collective intelligence mechanisms, establishing a working mechanism that allows listening and speaking, expanding the usual framework of work and investigating new possibilities and issues.

During this work process, some objectives were established, a working method was defined and a series of actions were proposed. This set of activities has been developed over time, documented and evaluated in a way that allowed redefining and adjusting what was initially thought. The intention has been to produce a specific and individualized result for Castelló, coming from the knowledge of the environment and strengthened by academic and professional knowledge. In addition, it is expected to function as a mechanism to eradicate violence against women.
\end{abstract}

Key words

Gender perspective; Urban Planning; Architecture

\section{Resumen}

En el trabajo abordado para la incorporación de la perspectiva de género en Castelló (Comunidad Valenciana, España), ha coincidido la circunstancia de un equipo técnico municipal excepcional, liderado por Consuelo Leal, y un equipo de gobierno político respetuoso con las decisiones y recomendaciones técnicas. El equipo asesor en cuestiones de género - redactor de este texto- ha contribuido, fundamentalmente, a que se pongan en marcha los mecanismos de inteligencia colectiva, estableciendo mecanismo de trabajo que permitieran escuchar y hablar, ampliar el marco de trabajo habitualmente empleado e indagar en nuevas posibilidades y cuestiones.

Durante este proceso de trabajo, se establecieron unos objetivos, se definió un método de trabajo y se propusieron una serie de acciones. Este conjunto de actividades ha sido desarrollado en el tiempo, documentado y evaluado de manera que se permitiera redefinir y ajustar lo inicialmente pensado. La intención ha sido producir un resultado específico e individualizado para Castelló, procedente del conocimiento del entorno y afianzado por el conocimiento académico y profesional. Y se espera que sirva como mecanismo para erradicar la violencia contra las mujeres.

\section{Palabras clave}

Perspectiva de género; Urbanismo; Arquitectura

1 Arquitecta; Profesora Contratada Doctora del Departamento de Proyectos Arquitectónicos de la Universitat Politècnica de Valencia (España). E-mail: ealvarez@pra.upv.es.

2 Arquitecto; Profesor Contratado Doctor del Departamento de Proyectos Arquitectónicos de la Universitat Politècnica de Valencia (España). E-mail: cjgomez@pra.upv.es. 


\section{Preámbulo}

Castelló es la capital de la provincia del mismo nombre, una de las tres provincias de la Comunitat Valenciana (España). Es una ciudad de tamaño intermedio en la que, según datos del INE (Instituto Nacional de estadística), ${ }^{3}$ viven 88.263 mujeres $(51 \%)$ y 83.406 hombres $(49 \%)$ y cuya área metropolitana ronda los 300.000 habitantes. Está ubicada en una zona llana, en la costa mediterránea española. El núcleo urbano que aloja el Ajuntament y otras instituciones está situado a unos cuatro kilómetros del mar, aunque existen otros núcleos urbanos, siendo el más destacado el del Grao, seguido del barrio de la Universitat Jaume I (conocida como UJI). La actividad económica local se desarrolla vinculada a la agricultura, la industria - principalmente la industria cerámica y una refinería del petróleo situada en el Grao-, la actividad comercial y el turismo, fundamentalmente de playa y sol. El análisis de los datos de población arroja un panorama similar al de otras ciudades de nuestro entorno en cuanto a distribución de edades, distribución de riqueza y acceso a oportunidades (ver el informe y la memoria justificativa del PGE). ${ }^{4}$

En cuanto a los datos oficiales de desempleo del municipio de Castellón de la Plana, en 2011 la tasa de desempleo era del 16\%, algo superior a la media de los valores provinciales y estatales de ese mismo año. A fecha de septiembre de 2017 la tasa de desempleo se sitúa en el $13,10 \%$, siendo significativo el mayor y creciente porcentaje de paro registrado por mujeres $(58,65 \%)$.

El PGOU (Plan General de Ordenación Urbana) de Castellón, redactado en el año 2000, fue anulado en 2008, cuya sentencia fue ratificada en 2012 por el Tribunal Supremo. En diciembre de 2013 se envía un borrador de Plan General a la Generalitat Valenciana que contesta en junio de 2014. En julio de 2015, además, entró en vigor la Ley 5/2015 de la Generalitat de Ordenación del Territorio, Urbanismo y Paisaje de la Comunidad Valenciana (habitualmente conocida por LOTUP) y que en el presente momento está en proceso de modificación y actualización, uno de cuyos objetivos es profundizar en la definición de mecanismos para la mejor incorporación de la perspectiva de género en el planeamiento urbano. Entre otras cosas, dicha Ley de 2015, elimina el tipo de documento PGOU y establece dos nuevos mecanismos de ordenación: el Plan General Estructural (PGE) y el Plan de Ordenación Pormenorizada (POP).

Desde finales de 2013, pues, el Ajuntament de Castelló está realizando las operaciones necesarias para subsanar la anulación judicial del documento de planeamiento urbano, teniendo, además, que ajustarlo a la nueva legislación. El equipo redactor de los nuevos documentos quiso incorporar la perspectiva de género al planeamiento, no sólo como indica en su articulado la LOTUP entonces vigente, sino de una manera claramente estructural y transversal, para lo cual contactó con los autores de este texto, solicitándoles, además, la redacción del Informe de Evaluación de Impacto de Género, documento que en aquel momento no era obligatorio (en la Comunidad Valenciana ha pasado a serlo a partir de diciembre de 2016).

3 INE, Instituto Nacional de Estadística, http://www.ine.es/.

4 Memoria Justificativa del PGE de Castelló en exposición pública en la web al siguiente enlace: https://s3-euwest-1.amazonaws.com/urbanismo/ MEMORIA+JUSTIFICATIVA.pdf.

\section{Planteamiento}

“¿Cómo sería una ciudad si no fuese sexista?” es la pregunta que se hacía la arquitecta feminista Dolores Hayden en 1980. En aquel artículo Hayden desgranaba cómo el entorno construido según el crite- 
rio predominante - negocio inmobiliario y beneficio económico seguro, directo y rápido-, contribuye muy poco a favorecer la vida de las personas que tienen que combinar trabajo remunerado y cuidado de otros, más aún si las condiciones sociales no son buenas. Desde este punto de vista, según la autora, vivienda asequible y cómoda, trabajo digno y adecuado, apoyo al cuidado y a la dependencia o movilidad sostenible... son aspiraciones difícilmente combinables en el entorno urbano y suburbano contemporáneo. Precisa aún más: son difícilmente combinables en el ámbito de la gestión propia privada y casi imposible de combinar si se espera que procedan de servicios y sistemas públicos.

Ya desde el mismo título del artículo, en el que emplea el término $s e-$ xista, Hayden establece el punto de vista que va a orientar su argumentación, ${ }^{5}$ de forma que ha abierto nuevas maneras de abordar las cuestiones urbanas y arquitectónicas, basadas en la perspectiva feminista.

El término sexista está muy bien explicado por Jo Freeman (1971), cuando expone que

Partiendo de la creencia tradicional de la diferencia entre sexos, el sexismo encarna dos conceptos centrales. El primero es que los hombres son más importantes que las mujeres. No necesariamente superiores — somos muy sofisticados hoy en día como para usar esos términos nocivos- sino más importantes, más significativos, más valiosos, más apreciados [...]. El primer concepto central del pensamiento sexista es que los hombres hacen el trabajo importante en el mundo y que el trabajo hecho por los hombres es lo que es importante (p. 8, traducido del inglés).

Si ligamos ambas reflexiones - Hayden y Freeman- podemos deducir que una ciudad sexista sería aquella que no considera importante el trabajo habitualmente realizado por las mujeres y, por ende, no lo identifica, no lo aborda, no lo proyecta... es decir, no le otorga el lugar y la calidad espacial que, desde el punto de vista de quienes sí realizan dichas tareas, requeriría.

Cuando a finales de 2015, surgió la posibilidad de colaborar con el equipo redactor del Plan General Estructural (PGE) de Castelló, liderado por la arquitecta municipal Consuelo Leal, para la incorporación de la perspectiva de género, una de las preguntas que pronto surgió fue ¿Cómo sería Castelló si todos los habitantes y sus tareas fueran tenidas en cuenta con el mismo interés?

Esta pregunta inicial acarreó otras preguntas enlazadas: ¿Es Castelló una ciudad sexista? ¿Cómo se compone la ciudadanía de Castelló? ¿Qué necesidades tienen las personas que viven en Castelló? ¿Cambiaría la ciudad si la perspectiva del personal técnico fuese feminista?

Dichas preguntas surgieron sobre la marcha del trabajo y, en cierta manera, gracias a que el equipo redactor era claramente paritario, y totalmente volcado en la aproximación disciplinar a la realidad cotidiana de las personas... pero, además, gracias a que el equipo de gobierno municipal, también paritario, apoyó y fomentó las iniciativas que fueron surgiendo durante el proceso de elaboración del PGE.

Feminismo, perspectiva de género, paridad, tareas del cuidado, inclusión, justicia... son términos y nociones que van constantemente vinculados y que, una vez asumidos, cuesta trabajo pensar que, en otros momentos, no hayan sido enfoques rotundamente prioritarios. Se desconoce si lo van a ser en el futuro. Lo que sí es innegable es que allí donde se tiene en cuenta a todo el mundo y sus necesidades vitales, el
5 Dolores Hayden es una arquitecta, historiadora, profesora universitaria, investigadora y poetisa feminista, con una larga e importante trayectoria crítica. Más información en https://undiaunaarquitecta.wordpress.com $/ 2015 / 07 / 26 /$ dolores-hayden-1945/. 
entorno social y urbano mejora como, en particular, demuestra el caso de la ciudad de Viena (cfr. Damyanovic, 2013, p. 17).

El PGE de Castelló ha aplicado este enfoque como acción prioritaria de todo el planeamiento, declarándolo literalmente desde la página 7 de la introducción de su memoria justificativa (Leal Jiménez, 2018). La tarea del equipo asesor en cuestión de perspectiva de género ha consistido en informar, apoyar y evaluar el proceso desde el inicio de la colaboración, entendiendo que el responsable de trasladar las intenciones a la realidad es el conjunto de todos los documentos del PGE y es, por tanto, el equipo redactor de dicho documento el que incorpora la perspectiva de género.

\section{Objetivos}

Las leyes a las que se sujeta el PGE de Castelló, acometen la búsqueda de Igualdad entre Mujeres y Hombres. A pesar de ello, tratan la desigualdad o igualdad de una manera abstracta y genérica.

Sin embargo, y como contrapunto, es sorprendente que en el informe realizado por el Observatorio de Igualdad de Género de América Latina y el Caribe en 2014, sobre cómo enfrentar la lacra de la violencia contra las mujeres (CEPAL, 2014), el primer capítulo esté dedicado a la autonomía de las mujeres, donde se indica que,

En parte, esta idea de igualdad también es producto de la interpelación desde las reflexiones y demandas del feminismo, que han puesto sobre el tapete diversos aspectos vinculados a la desigualdad de género y la discriminación de las mujeres. Se ha llegado así al convencimiento de que la meta de la igualdad no se alcanzará mientras las mujeres no posean autonomía en todos los ámbitos. La autonomía es definida como "el grado de libertad que una mujer tiene para poder actuar de acuerdo con su elección y no con la de otros. En ese sentido, existe una estrecha relación entre la adquisición de autonomía de las mujeres y los espacios de poder que puedan instituir, tanto individual como colectivamente" (Naciones Unidas, 2005, pág. 114).

La igualdad y el pleno ejercicio de los derechos humanos de las mujeres tienen como condición la autonomía de las mujeres en la vida privada y pública. En virtud de esta premisa, el Observatorio de Igualdad de Género de América Latina y el Caribe viene trabajando desde 2009 en tres pilares que dan cuenta de distintos aspectos de la autonomía relacionados con la capacidad para generar ingresos propios y controlar los activos (autonomía económica), con el control sobre el propio cuerpo (autonomía física) y con la plena participación en la toma de decisiones que afectan su vida y colectividad (autonomía en la toma de decisiones) (p. 11).

El PGE de Castelló expresa su interés por traducir dicha búsqueda de autonomía a la esfera del urbanismo. Por lo que al documento respecta, se ha intentado observar cómo la ciudad y su configuración contribuyen a la autonomía de las mujeres. Este objetivo tan específico, exigía, en primer lugar, saber cuál es la situación real y actual de las mujeres respecto de dichas cuestiones y en su comparación a la situación equivalente de los hombres. Y, en segundo lugar, obligaba a que las decisiones tomadas vinieran mediatizadas por una serie de indicadores y procesos de medición de resultados que permitiese evaluar el avance en el establecimiento de las medidas o los logros obtenidos a lo largo 
del desarrollo o implantación de las determinaciones del presente plan general estructural.

Para conocer cómo otras personas necesitan usar la ciudad y sus sistemas, hay que preguntar. Hay que preguntar muchas veces, a muchas personas y de manera correcta y solvente, si esos datos deben de servir para reconducir el diseño de la ciudad. Es por ello que los mecanismos de participación y toma de datos deben perfeccionarse y diversificarse. Es preciso, también, bucear en la enorme cantidad de bases de datos existentes y ver qué datos están desagregados por sexo biológico, en primera instancia.

Hay que ver para resolver, transformar las necesidades en proyectos, por seguir la consigna expresada por el grupo de investigación y trabajo Proyecto Habitar de Buenos Aires, liderado por Eugenia Jaime y Clara Mansueto (2012).

[Ellas] Progresivamente, y con respeto al lector, aportan información re-construida con la población. Pinceladas de la historia, el arte y los sentimientos producidos por experiencias vividas en los barrios populares son los insumos para entender cómo se fueron configurando esos espacios calificados como: 'invisibles (espacios 'verdes' ocupados), 'criminalizados' (ilegales), 'negados' (irregulares), 'degradados' (insalubres, precarios, en riesgo, muertos, a 'remediar', a 'renovar') etc. La forma de trabajo participativa e intersectorial que proponen permite entender estas urbanizaciones desde una visión diferente al enfoque tecnocrático positivista o de negocio urbano inmobiliario (Enet, 2012, p. 4).

Esa información re-construida junto a los que viven el espacio construido es difícil de obtener pues no se reduce sólo a estudios estadísticos o a elaboración de estudios de actores singulares. Tal y como indica Arendt (2008), se requiere un interés adicional por compartir y comprender, por aproximarse a la realidad y no despegarse de la misma.

Cuando se separa totalmente una idea de su base en la experiencia real, no es difícil establecer una conexión entre ella y casi cualquier otra idea. En otras palabras, si presuponemos que existe algo así como una esfera independiente de puras ideas, todas las nociones y conceptos no pueden sino estar relacionados, porque todos deberían su origen a la misma fuente: la mente humana en su extrema subjetividad, no afectada por la experiencia y sin ninguna relación con el mundo (p. 53).

\section{Método}

Esta aproximación a la realidad diversa, es lo que el equipo redactor y asesor del PGE ha considerado incorporación de la perspectiva de género en la construcción del entorno colectivo mediante el planeamiento urbano, y supone la aplicación de un método de trabajo interactivo que ha sido aplicado por un equipo previamente entrenado en mirar y ver la realidad compleja desde el punto de vista de todas las personas usuarias y, en particular, desde el punto de vista de las mujeres. La perspectiva de género no constituye un elenco de soluciones a posteriori que resuelvan una situación ya originada, sino que, ante una situación a estudiar, supone y plantea una aproximación metodológica diferente. Para ello, ha re-construido la información en colaboración con la población (Mansueto y Jaime, 2012) dando validez al conocimiento no suficien- 
temente acreditado pero existente entre los usuarios. También ha reconocido el valor de uso y la diversidad de sus patrones de actividad y valorado el uso colectivo de los espacios públicos y privados.

Por otra parte, ha tratado de entender que la sostenibilidad económica debe añadir a sus variables de estudio aquellas que afectan al trabajo no remunerado del cuidado, ya que es una realidad paralela no contabilizada y que, por tanto, supone una distorsión de dicha realidad. Y ha tratado de entender que la gestión del tiempo personal y colectivo es una variable fundamental a tener en cuenta en el diseño urbano. Tiempo, memoria y comportamiento son aspectos vinculados al entorno construido que pueden ser observados con perspectiva de género, ya que se manifiestan de manera diferente para cada persona y situación.

Además, se han planteado actuaciones y proyectos específicos y particularizados mediante datos e información obtenida tanto participativa y colaborativamente, así como del análisis de bases de datos ya existentes, mezclando diversos patrones de uso (Venturi y Scott Brown, 2004) y ha activado actuaciones concretas -en el entorno construidoenfocadas en aumentar la autonomía de las mujeres como, por ejemplo, para incrementar la:

1. Autonomía económica de las mujeres: con decisiones que contribuyan a la gestión de las oportunidades específicas de trabajo, movilidad, formación, educación, camino escolar, cuidado de la dependencia...

2. Autonomía física de las mujeres: con actuaciones que contribuyan al acceso a la vivienda digna, zonas verdes próximas, control de la contaminación, accesibilidad, seguridad, percepción de seguridad, limpieza y mantenimiento, baños públicos, servicios de proximidad, acceso a la salud y al deporte, acceso a comida no contaminada y a precio asequible...

3. Autonomía de las mujeres en la toma de decisiones: insistiendo en el paisaje urbano como historia pública (Hayden, 1995), con el aumento de la legibilidad y orientación urbanas, la distribución de servicios en el territorio, accesibilidad, movilidad, limpieza y decoro, seguridad...

También se han definido indicadores cuantificables que permitan verificar cuantitativamente el progreso en la incorporación de la perspectiva de género en el entorno construido de Castelló. Más aún, se han vinculado el avance de los indicadores generales del PGE a aspectos asociables a criterios de perspectiva de género.

$\mathrm{Y}$, finalmente, ha sido comunicado públicamente para que se comprenda que las soluciones enfocadas hacia la disminución de la desigualdad de las mujeres, sin duda, son mejores para el conjunto de la sociedad y del entorno construido. No sólo por una cuestión de justicia —que es la razón principal—, sino por una cuestión de pragmatismo, ya que mejoran la vida de todos los colectivos.

\section{Acciones}

Como ya se ha indicado, la perspectiva de género debe disolverse en las determinaciones del PGE, siendo el desarrollo y la aplicación del PGE a lo largo del tiempo lo que incorporará la perspectiva de género efec- 
tivamente en el entorno urbano de Castelló. Por tanto, la perspectiva de género dependerá de lo que cada persona entienda que es, en el ámbito de su esfera de trabajo. Y, por supuesto, no es un aditamento de última hora. El equipo redactor del PGE de Castelló ya estaba sensibilizado por el tema, así como el equipo de gobierno municipal. Sin embargo, demandaban información específica de cómo proceder. Para ello el equipo asesor, promovió varias acciones, las documentó y las evaluó. Describimos dichas acciones a continuación:

ACCIÓN 01: Informar sobre los objetivos 'tipo' generales a los que puede aspirar la incorporación de la perspectiva de género en el PGE de Castelló, lo que supuso la revisión del conjunto de la producción académica e investigadora sobre cómo incorporar la perspectiva de género en el entorno construido, y revisión del conjunto de documentos que habilitan su transferencia a la realidad por parte de las instituciones - en particular por parte de Ayuntamientos-, lo cual configura un 'estado de la cuestión' que, una vez revisado, permita definir el marco en el que se insertan las decisiones propuestas en este PGE. Entre las actuaciones revisadas destacan por su importancia:

- La incorporación de la perspectiva de género en la ciudad de Viena: desde 1991, la ciudad de Viena realiza una acción decidida, dirigida a la incorporación de la perspectiva de género en todas las decisiones y actuaciones que dependen de esta entidad. Dicha actividad ha sido liderada por Eva Kail, urbanista feminista (Damyanovic, 2013) que dirigió la primera oficina dedicada a estos asuntos, insertada dentro de la administración municipal. A lo largo de los últimos 20 años, el ayuntamiento de Viena ha desarrollado políticas de género en el ámbito de la vivienda, del espacio público, de la movilidad peatonal, del transporte público, etc. En la actualidad, lidera un proyecto piloto para construir 800 viviendas, experimentando con nuevos modos de participación. El trabajo realizado por Eva Kail en Viena ha sido difundido en diversas acciones llevadas a cabo en Valencia vinculadas a la Universitat Politécnica de Valencia, la Universitat de València y la Generalitat Valenciana, como puedan ser: la organización de sesiones de divulgación —que contó con la presencia de Eva Kail- organizadas por la UPV en $2015 ;{ }^{6}$ las Jornadas de Género y Política Urbana en julio de 2016 organizadas por la GVA y el IVE, ${ }^{7}$ que también contó con la presencia de Inés Sánchez de Madariaga y Jordi Bosch, entre otros (Serrano Lanzarote, B., Mateo Cecilia, C. y Rubio Garrido, A., 2017); el Foro FemUrbs, en septiembre de 2016, organizado por la Universitat de València, en el que participó Adriana Ciccoleto de Col.lectiu Punt 6, además de Eva Kail, en una nueva visita a Valencia. El Ayuntamiento de Viena y la Universidad BOKU han publicado un libro - ya mencionado- de recomendaciones y buenas prácticas (Damyanovic, 2013) que recoge organizadamente las tareas realizadas en la ciudad de Viena para incorporar la perspectiva de género.

- Las Directrices de Ordenación del País Vasco, trabajo realizado por Inés Sánchez de Madariaga y sus colaboradoras en 2015, en el que se proponen Medidas básicas y Acciones de gran impacto, insistiendo en la selección de objetivos estratégicos para procurar la incorporación efectiva de la perspectiva de género en el planeamiento urbano que se lleve a cabo en el País Vasco. Lo más
6 La Conferencia es accesible online: https://media.upv.es/\#/por$\mathrm{tal} /$ video/31f9194d-bd5b-4b64-9471$7 \mathrm{e} 48 \mathrm{c} 2147673$.

7 La Conferencia es accesible online: https://www.youtube.com/playlist?list=PL VG7IkLJIJ5jklxQBEaHk-RP7id7GrCFA. 
destacable de este trabajo es que se presenta como una estrategia y un proceso planificado, no como objetivos puntuales.

- La actividad del Col.lectiu Punt 6 y de Zaida Muxí: Col.lectiu Punt 6, es un colectivo de arquitectas y sociólogas radicado en Barcelona y en cuya fundación tuvo un papel fundamental Zaida Muxí. A lo largo de su existencia han investigado - e investigan- sobre cómo re-construir la información acumulada en las personas usuarias y cómo transferirla a los documentos de planeamiento (Col.lectiu Punt 6, 2017). En enero de 2017, varios miembros de dicho colectivo participaron en el congreso MORE que tuvo lugar en Florencia, donde se exponía cómo se incorporaba la perspectiva de género en distintas facetas de la investigación y del trabajo. En dicho congreso destacó la participación de Liza Fior del colectivo $m u f,{ }^{8}$ cuyo planteamiento directo es que el hecho de aplicar la perspectiva de género supone desarrollar proyectos particularizados y específicos, ajustados a las necesidades bien definidas de colectivos bien definidos.

- El PGOU Irún y PGOU Bilbao: estas dos ciudades han incorporado la perspectiva de género en sus documentos de una manera bastante clara y transmisible. Ambas tareas han sido dirigidas por los arquitectos de ProjektaUrbes, Miren Vives y Patxi Galarraga, $y$, aunque cada documento es diferente, en ambos se pone de manifiesto la necesidad de conocer la realidad de una manera pormenorizada (Vives, 2014). Además, en la actualidad, están incorporando aspectos relativos al envejecimiento activo y las condiciones de salud que el medio urbano proporciona, como la calidad del aire o la cercanía de zonas verdes, tal y como explicaron en su visita a Valencia en noviembre de 2017. ${ }^{9}$

- SET para introducir la perspectiva de género en el proceso urbano: la Generalitat Valenciana, en diciembre de 2016, lanza una recopilación en materia de arquitectura y urbanismo desde la perspectiva de género en la que organiza la información en siete grandes grupos de temas internacionalmente acreditados como temas de fondo a tratar para propiciar la incorporación de la perspectiva de género en el entorno construido: Modelo de ciudad, Percepción de seguridad, Espacios públicos y accesibilidad, Movilidad, Vivienda, Representatividad y señalización urbana y Participación ciudadana Valenciana (Azara Escrivá y Gil Vila, 2016).

ACCIÓN 02: Debatir con los responsables del equipo redactor, la selección de objetivos específicos a aplicar en el PGE de Castelló, con la finalidad de incorporar la perspectiva de género en las actuaciones previstas en dicho documento.

A partir de julio de 2016 se producen reuniones preliminares con Consuelo Leal y Fernando Calduch para enfocar la manera en que se va incorporar la perspectiva de género en el PGE. Se establece la necesidad de analizar el plan anulado; se recomienda una contextualización de la situación socioeconómica actual y se propone definir un listado de recomendaciones y de objetivos relativos a la perspectiva de género a incorporar por el nuevo PGE.

Entre septiembre y octubre de 2016 se analizan los documentos e información existente, en particular los documentos del PGOU de los años 2012 y 2000, en los que, una vez revisados, destaca la ausencia de criterios explícitos de análisis demográfico de la población por edad 
y género o de su situación socioeconómica pormenorizada; así como la ausencia de criterios que pretendan disminuir la desigualdad o, al menos, contribuir a que no aumente. Destaca de dicho documento, la proporción de páginas dedicadas al estudio de viabilidad socioeconómica, en relación al conjunto de páginas del documento. También se revisa la publicación editada después de la celebración de varias reuniones sectoriales - foro técnico, foro de infraestructuras estratégicas, foro de dinamización económica, foro de calidad de vida, foro de sostenibilidad-conocidas como Foros de Urbanismo de Castelló (Ayuntamiento de Castellón de la Plana, 2014), documento que acopia los debates llevados a cabo en Castelló.

Ante la situación detectada, en la que se asumía con naturalidad que la perspectiva de género podría ser un aditamento a ser añadido al final de un proceso más largo e importante, en noviembre de 2016 el equipo asesor de género redacta un documento titulado 'Perspectiva de género: Recomendaciones para su incorporación efectiva en el planeamiento urbano de Castelló 2017' donde se indica que la aplicación de la perspectiva de género supone asumir un método de trabajo sostenido en el tiempo. Dicho método no produce resultados inequívocos y simples; al contrario, asume la necesaria complejidad en la que se desenvuelve la vida colectiva, tratando de aproximarse a quienes muestren mayor necesidad o debilidad.

Por tanto, fue necesario la provisión de datos estadísticos desagregados y eficientes de amplio espectro sociológico y económico, desagregados por género, y se consideró de interés realizar un trabajo colaborativo con sociólogos y trabajadores sociales para conocer la realidad que las estadísticas no pueden mostrar. Se consideró indispensable la organización de procesos participativos in situ y online que recogiese datos para conocer la situación de partida, pero, también, que recogiesen el modelo a futuro que los distintos colectivos desean o imaginan, para lo cual se propone la revisión del modelo propuesto en el decálogo a futuro para evaluar su impacto en relación a las tareas de cuidado y resto de tareas cotidianas.

Por otra parte, también se vio la necesidad de realizar talleres con los responsables de las distintas áreas de trabajo dentro de la administración municipal — vivienda, movilidad, espacio público, seguridad, servicios sociales, etc.- para dar a conocer la perspectiva de género, sus objetivos y sus métodos; así como se vio la necesidad de realizar seminarios de difusión de debate sobre criterios y métodos de trabajo entre el personal técnico redactor del plan.

También se recomendó que los equipos redactores de los distintos documentos del plan fuesen paritarios, ya que es un modo natural de incorporar objetivos asociados a la vida diaria. Y el empleo de un lenguaje inclusivo y sencillo en todos los documentos redactados desde la Unidad Técnica del Ajuntament.

El 15 de noviembre 2016, se mantuvo una reunión en el Ajuntament de Castelló con los responsables del equipo redactor, para comentar y debatir los temas propuestos en dicho informe, y se acordó el siguiente listado de actividades:

- La incorporación de los redactores de esta memoria de género a los procesos participativos previstos por el Ajuntament que comenzarían en diciembre 2016 (17 sesiones) con la finalidad de dar a conocer los objetivos del planeamiento e invitar a los habitantes a hacer aportaciones, preguntas, sugerencias y críticas es- 
pecíficas. Se adecuó el horario inicialmente propuesto, de manera que se flexibilizase y se facilitase la asistencia de distintos colectivos.

- Ante la imposibilidad de propiciar un proceso de toma de datos participativo y colaborativo en todo Castelló, se propuso desarrollar un programa de actividades más amplio y tomar datos en una zona 'piloto' cuyos datos pudiesen servir de referencia teórica a otras zonas de Castelló. La zona elegida fue la zona de la Avda. Valencia/Gran Vía, al sur de Castelló.

- La elaboración de una encuesta online específica sobre cuestiones relativas al género y la vida cotidiana. Se acompañaría de una versión en papel a repartir en las distintas sesiones, de manera que se superase la 'brecha digital'.

- La organización de sesiones de difusión y formación en cuestiones de perspectiva de género del personal administrativo y técnico vinculado al Ajuntament de Castelló.

- La elaboración de una guía de buenas prácticas y recomendaciones básicas.

- La decisión de elaborar el listado de objetivos específicos para incorporar la perspectiva de género en Castelló - a partir de dichos procesos de participación y formación-, de manera que esta perspectiva quede incorporada efectivamente y específicamente dentro de la multitud de documentos que formalizan un PGE.

- La presente memoria es sólo un índice o recuerdo de cómo ha sucedido esto; en realidad, sólo se conocerá la validez de los resultados, una vez evaluados los indicadores propuestos.

ACCIÓN 03: Impulsar la recogida de datos desagregados y específicos para Castelló, desde el punto de vista de género. Una vez decididos los objetivos anteriores, se realizaron diversas tareas cuya misión fue evidenciar la necesidad de conocer la realidad compleja de una manera específica y detallada. Para ello, se impulsó y animó a los servicios y equipos implicados a la recogida de datos desagregados y pormenorizados que reflejen la situación específica de cada grupo o colectivos. Reseñamos algunas de las tareas realizadas o animadas por este equipo asesor:

- Asistencia de los autores del informe -y de este texto- a varias de las jornadas participativas de divulgación del Plan General ${ }^{10}$ organizadas por el Ajuntament para evaluarlas y observar resultados. Dichas reuniones ya vieron modificado su calendario y horario para incluir cuestiones de género. Se diversificó la oferta de días, decidiéndose jueves tarde y sábado mañana; se modificó el horario pasando a $18 \mathrm{~h}$ en vez de $19 \mathrm{~h}$ para que pudieran asistir las personas que cuidan niños pequeños; y se dispusieron zona de juegos infantiles vigiladas para que los niños y niñas pudieran entretenerse, mientras sus familiares atendían a la reunión, especialmente la mañana del sábado.

- El arquitecto colaborador Xavi Aragó recogió más de 150 referencias en prensa como consecuencia de las jornadas participativas. Se ordenaron y se pusieron a disposición del equipo redactor, con la finalidad de observar patrones de uso desde el punto de vista del género. El análisis minucioso de dichas referencias de prensa resaltó el interés general recogido por los periodistas.

talle en el siguiente enlace: http:/ en deplageneralcastello.es/participacio\# [último acceso realizado el 29/03/2018]. 
- Se revisó la documentación publicada en la web específica para el Plan General alojada en la web del Ajuntament de Castelló, que lleva por título Pla General \#CastellóCiutatViva y en la que puede accederse a diversos documentos del plan. ${ }^{11}$

- Se diseñó y publicó un formulario específico o encuesta relativa a la incorporación de la perspectiva de género 'castelló(n) suma'12 para conocer la situación actual de la ciudadanía en Castelló y para conocer las necesidades que querrían solventar mediante la aplicación del plan general.

- Entre las acciones piloto, encaminadas a obtener una información más ajustada a la realidad, se propuso un Paseo de Jane en la zona Avenida de Valencia. El paseo de Jane es una actividad participativa que se celebra en todo el mundo desde el año 2009, y que tiene como misión ver el entorno construido con 'otros ojos', es decir con los ojos de las actividades cotidianas y que busca mantener y conocer la memoria de los lugares, en la línea propuesta por Hayden en Los Angeles (Hayden, 1995) como historia pública, y permitir que la gente se conozca y se dé cuenta de que comparten problemas comunes, en la línea de los rap groups descritos por Freeman (Freeman, 1971). Como material de difusión y preparación, se diseñó un cartel, se facilitó un plano interactivo descargable en el móvil, una encuesta en papel y online preguntando por aspectos adaptados a esa zona de Castelló. El 20 de mayo de 2017 se llevó a cabo el paseo al que asistieron una docena de personas y varios estudiantes de la UPV -que documentaron el paseo. En dicho paseo se puso de manifiesto la escasa memoria de las mujeres, existente en el callejero o monumentos de la ciudad, a pesar de la excepción que supone tener en la zona el colegio Matilde Salvador. El recorrido previsto, de 1,8 km aproximadamente, se realizó a pie, duró desde las 10 am hasta las $2.30 \mathrm{pm}$; y tuvo 7 puntos de parada y charla.

- Exposición de los trabajos realizados por los alumnos del Taller 1 de la Escuela de Arquitectura de la Universitat Politècnica de València, que estudiaron el entorno de la Crèmor. Dicho trabajo, se expuso en la Escuela de Arquitectura con motivo de la visita del Presidente de la Generalitat Valenciana y la alcaldesa de Castelló a la UPV, el 28 de noviembre de 2016; y entre el 7 de abril y el 10 de mayo de 2017 se expuso en el Menador Espai Cultural de Castelló, donde recibió la visita de parte de la corporación municipal, del equipo redactor del PGE y de las asociaciones de vecinos de la zona. Durante todas estas sesiones -incluidas las sesiones de trabajo de los estudiantes- los vecinos expusieron sus necesidades y aspiraciones, destacando la petición recurrente de mejora del espacio público y zonas verdes: iluminación, equipamientos y la dotación de conexión con el resto de la ciudad.

ACCIÓN 04: Participar en la construcción y definición posible de objetivos específicos del PGE, vinculados a la demanda y carencias detectadas, que vayan encaminados a la incorporación de la perspectiva de género en el PGE de Castelló. Para ello se asesoró a los distintos equipos redactores del PGE y de sus Estudios anexos y a los distintos estamentos administrativos y políticos, actuando y trabajando en el Ajuntament de Castelló, de manera que pudiesen manejar una idea consensuada, ampliada y coherente de lo que supone la incorporación de la perspectiva de género en el planeamiento urbano. Para ello se proponen varios
11 Cfr. http://www.plageneralcastello.es/inici [último acceso realizado el 29/03/2018].

12 Se publicó una encuesta online, vinculada a la web del Ajuntament y que es consultable en el link: https://goo.gl/ yFZjQi. 
mecanismos de trabajo que ya habían sido indicados en el informe preliminar, entre los que destacamos:

- Jornada de divulgación y formación del personal del Ajuntament, que tuvo lugar el día 6 de julio de 2017, entre las 9.30 y las $14.30 \mathrm{~h}$, en las dependencias municipales contando con gran asistencia. La sesión se denominó 'Castelló: Criterios para la aplicación de la perspectiva de género’ y tuvo dos módulos que se repitieron para que los distintos servicios no se quedaran desatendidos (lo cual es en sí una cuestión relativa a la incorporación de la perspectiva de género).

- Reuniones de revisión de criterios y documentación del equipo redactor y de revisión de documentación realizada por equipos externos al Ajuntament. A partir de junio de 2017 se decidió realizar reuniones sistemáticas de revisión de documentación. A dichas reuniones acudirá el equipo redactor municipal y el equipo asesor. La finalidad de dichas reuniones será revisar la documentación en marcha, discutir los criterios y objetivos sobre los que estén trabajando y clarificar dudas o discusiones que pudieran plantearse durante la redacción de las distintas partes de los distintos documentos del plan.

ACCIÓN 05: Selección y priorización de objetivos particularizados y adaptados a la situación detectada en Castelló. Una vez revisado el estado de la cuestión y con los datos e informaciones disponibles en el caso de Castelló, se propone al equipo redactor estructurar los objetivos generales en varias líneas temáticas:

- Sobre el modelo urbano se decide que se debe lograr que el planeamiento atienda a las necesidades de todo el término municipal de Castelló (centro y barrios periféricos) y moderar el crecimiento urbano procurando la continuidad urbana dentro de los límites establecidos.

- Respecto de las infraestructuras se sugiere el priorizar la atención a las cuestiones de saneamiento y alumbrado, adecuándolas a los diferentes ámbitos del término municipal.

- En relación a los usos, se propone establecer la compatibilidad y la mezcla de usos procurando que la ciudad se mantenga relativamente activa todo el año; distribuir los servicios administrativos y sociales por todo el ámbito de planificación; promocionar, claramente, los usos que facilitan el cuidado. También se propone el distribuir las zonas de vivienda en todo el ámbito de actuación teniendo en cuenta los requisitos dotacionales inmediatos necesarios, dotando de equipamiento vinculado al cuidado en todas las zonas de Castelló.

- En lo relativo al espacio público y la movilidad se propone el establecer redes de pequeñas zonas verdes y caminos verdes en todo el ámbito de planificación; entrelazar la movilidad peatonal con la ciclista y la rodada, de modo que se favorezca todo tipo de recorridos según grupos de edad y circunstancia; equipar dichos recorridos con facilidades como servicios higiénicos, bancos para descansar o fuentes de agua potable; procurar la accesibilidad universal; diseñar los espacios públicos de manera que sean flexibles en su uso y permitan usos no previstos (bailar, jugar, actuaciones musicales); y el ofrecer equipamientos y zonas 
de ocio particularizado a todos los grupos de habitantes (gente joven, gente mayor, inmigrantes).

- Sobre la sensación de seguridad se promoverá la mezcla de usos para garantizar la presencia continuada de personas en las calles; se garantizará la visibilidad en la vía pública y procurar recorridos alternativos; y se incentivará la legibilidad y orientación urbana.

- En cuanto a la memoria y a la historia pública, se propone reforzar la memoria de las mujeres en la ciudad y el dignificar el espacio público previo a los edificios donde tradicionalmente trabajaban o trabajan las mujeres (colegios, mercados, hospitales, peluquerías, tiendas).

- Respecto a la participación ciudadana, se propone visibilizar el que sí se tiene en cuenta, atendiendo y comunicando las cuestiones surgidas a lo largo de las diferentes fases del proceso participativo, llevado a cabo en Castelló durante este tiempo.

ACCIÓN 06: Redacción de documentos por parte del equipo asesor para documentar las tareas ya acciones llevadas a cabo, sus conclusiones y resultados parciales y, finalmente, la redacción del informe de evaluación de impacto de género.

\section{Conclusiones}

Durante este proceso de trabajo, se establecieron unos objetivos, se definió un método de trabajo y se propusieron una serie de acciones. Este conjunto de actividades ha sido desarrollado en el tiempo, documentado y evaluado de manera que se permitiera redefinir y ajustar lo inicialmente pensado. La intención ha sido producir un resultado específico e individualizado para Castelló, procedente del conocimiento del entorno y afianzado por el conocimiento académico y profesional.

Una vez finalizado el proceso y elaborada la documentación —que proseguirá los pasos marcados por el proceso administrativo prestablecido- se destacan algunos hallazgos o conclusiones que pueden ser considerados singulares:

- Es necesario que el urbanismo contribuya a la ganancia de autonomía personal de las mujeres lo cual, por otra parte, supone el recuperar el sentido colectivo de la ciudad. El diseño del entorno urbano, los edificios y los espacios intermedios, la movilidad y la oportunidad, la capacidad para generar y disponer de recursos económicos... son cuestiones básicas que afectan a cómo las personas usan la ciudad y ejercen una influencia decisiva en la ganancia de autonomía, en particular, de las mujeres. Si se añade a esto la existencia de estudios que vinculan la ganancia de autonomía personal a la lucha contra la violencia machista, se entiende que el diseño de la ciudad puede y debe contribuir a la disminución de la violencia estructural contra las mujeres. Durante años, los autores de este trabajo pensaron que el diseño urbano tenía sus propios problemas disciplinares y que la violencia contra las mujeres era otro problema, de otra índole, que a veces sucedía en el marco de la ciudad. Sin embargo, mediante los procesos participativos se ha observado y comprobado cómo la configuración del entorno construido, tal y como la entendemos en la 
13 Expresión escuchada en una conferencia en la ETS de Arquitectura de Valencia en 2016, con motivo de unas jornadas de género y arquitectura.

14 Noción elaborada por los autores de este texto a raíz de un texto para un simposio organizado por IAWA (International Archie of Women Architects en la Universidad Virginia Tech) en 2018.

15 En la Conferencia celebrada en la ETSA de Valencia, ya mencionada anteriormente. actualidad, es un elemento más -e incluso un sujeto más- que ejerce violencia contra las mujeres: se puede considerar violencia el no poder acceder a un trabajo bien remunerado por falta de transporte público; o el no poder acceder a la educación o a la salud por no disponer de infraestructuras del cuidado...y así un largo, etcétera.

- Si establecemos, pues, un vínculo entre violencia, urbanismo y arquitectura, cobra interés el hecho constatado de que las mujeres pueden sufrir un continuum de violencia: ${ }^{13}$ violencia doméstica, violencia laboral, violencia en el entorno urbano, violencia institucional... Desde este punto de vista, y como contrapunto, en este trabajo se propone y busca el desarrollar la noción de continuum espacial, ${ }^{14}$ que promueve el romper el aislamiento que pueda producir el continuum de violencia y que se base en la recuperación del espacio público y su uso colectivo. Por tanto, la dimensión y configuración de ese espacio continuo, espacio de transición desde la vivienda al espacio urbano en sus distintas escalas, cobra relevancia y debe ser atendido específicamente, como instrumento sustancial en la erradicación de la violencia contra las mujeres.

- La siguiente cuestión detectada es que esa continuidad espacial demanda requisitos diferentes según sea la persona usuaria, sus costumbres y sus condiciones. Surge así la necesidad de detectar colectivos, grupos, condiciones, circunstancias... para poder ofrecer soluciones específicas y conducentes a la ganancia de autonomía personal. Es por ello que a lo largo del trabajo se ha hablado de la necesidad de conocer el diagrama detallado de la composición social desagregada de la población y los patrones de uso de cada grupo, de manera que la organización espacial contribuya a la mejora de las condiciones de partida de los habitantes. El trabajo con personal experto en sociología y el empleo de estadísticas desagregadas es esencial.

- A raíz de los procesos participativos llevados a cabo y ante el probable caso de conflicto de intereses, que pudiera producirse entre distintos patrones de uso, se sugiere que se disponga del conocimiento sobre qué colectivo o grupo dispone de menos recursos alternativos para resolver la situación originada, en caso de que no se aplicara la medida que pudiera producir dicho conflicto. Esto lleva a disponer de esquemas de patrones de usos valorados que puedan servir para priorizar y argumentar decisiones ante situaciones conflictivas. Tal y como Eva Kail indica constantemente, ${ }^{15}$ la perspectiva de género es una herramienta para priorizar decisiones.

- Se destaca la necesidad de profundizar más en los métodos adecuados para canalizar los procesos participativos y en la aproximación al concepto de inteligencia colectiva, tan necesarios para poder abordar un fenómeno tan complejo como es el diseño del marco físico y del uso de nuestro entorno construido.

- Este tipo de información es difícil de obtener por procesos participativos puntuales, que si se generalizaran tenderían a cansar a la población, que, por otra parte, parcialmente, podría no ver satisfechas sus indicaciones o demandas. Es por ello, que se entiende que se debería tender a tener mecanismos de participación e información continuos, a modo de observatorios, gestionados por personal adecuado y específico, que permita disponer 
de información detallada y valorada en todo momento de todo el entorno urbano, y que permita priorizar decisiones tanto en el ámbito de la política como del urbanismo y la edificación. Es decir, más que iniciar procesos participativos puntuales para cada ocasión, se propone que haya un proceso participativo continuo, siempre en marcha, interactivo que esté recogiendo datos y expectativas de una manera continua y autónoma y que refleje los procesos de inteligencia colectiva que puedan surgir.

- Si se desea tener una imagen diversificada y valorada del uso que las personas hacemos de la ciudad o entorno construido, se entiende que los procesos de participación son necesarios en todas las fases de decisión: en la de análisis y detección de necesidades; en la de diseño y proyección de futuro, ya que cada grupo o colectivo puede tener expectativas o intenciones diferentes; y también en el proceso de evaluación de resultados, ya que el resultado de las acciones determinadas puede afectar a los habitantes y personas usuarias de maneras distintas. Por tanto, el observatorio continuo y estable propuesto debería recoger datos en las diferentes fases de interacción: sobre qué hace falta y para quien; qué se desea tener y por qué; y sobre cómo cada actuación afecta a los distintos grupos de personas usuarias.

- A lo largo de este texto, se ha hecho hincapié en que la perspectiva de género, básicamente, supone un método diferente de abordar la realidad consistente en una reconstrucción colectiva de la realidad, en un diseño específico que promueva la continuidad espacial según patrones de uso valorados y en un proceso continuo de evaluación y revisión de validez de resultados.

- Desde este punto de vista, cobra enorme importancia la definición de los indicadores de evaluación de resultados. A lo largo de esta experiencia en Castelló se ha entendido que más que disponer de indicadores específicos y particulares de evaluación de impacto de género - susceptibles de desarrollarse o no-cualquier indicador deberá estar definido, de manera que, si se verifica el indicador en cuestión, la parte de incorporación de perspectiva de género que le corresponda, se verificará. Este modo de proceder supone el rediseñar todos los indicadores que habitualmente se emplean de manera que el desarrollo en el tiempo de dichos indicadores, garanticen la necesaria incorporación de la perspectiva transversal de género. Para ello, se ha estado trabajando colaborativamente con el personal técnico del servicio de urbanismo de Castelló, con el servicio de urbanismo de Valencia y con grupos de trabajo de Madrid, proceso que aún continúa.

- Se ha visto la necesidad de disponer de guías y recursos organizados, que ayuden a los servicios técnicos municipales y a los equipos políticos de gobierno a plantear y abordar cómo se podría incorporar la perspectiva de género en el planeamiento y en la edificación. Existen distintas guías y textos - ya mencionados- ${ }^{16}$ pero sería necesario actualizarlos y ajustarlos a la legislación y realidad actuales y, sobre todo, configurarlos de manera interactiva y dinámica.

- También se ha visto el interés que podría tener el que la prensa diaria y los medios de difusión tuvieran una visión más precisa de lo que supone la incorporación de la perspectiva de género en la arquitectura y el urbanismo y contribuyeran a difundirla entre la población. Este proceso de difusión podría venir aparejado a
16 El más reciente es el SET usado para introducir la perspectiva de género en el urbanismo de la Generalitat Valenciana (Azara Escrivá y Gil Vila, 2016). 
los mecanismos de participación continua ya mencionados, pero este punto habría que precisarlo mejor.

- Es destacable también el que la demanda que más veces surgió en las reuniones y encuentros con colectivos fuera la necesidad de mantenimiento y calidad del espacio colectivo, así como la insuficiencia en la dotación de infraestructuras de saneamiento y de iluminación eléctrica. Y, aunque el mantenimiento no es estrictamente un tema de planeamiento urbano, sí que es consecuencia de este: los solares que quedan abandonados por gestión casi imposible, la ausencia de infraestructuras básicas, la falta de mezcla de usos que dinamicen y alarguen en el tiempo el uso de un espacio público... son condiciones que vienen propiciadas por el planeamiento urbano. Se propone que el diseño de la ciudad valore y evalúe qué tipo de mantenimiento va a tener un lugar y que, en caso de conflicto de intereses, priorice actuaciones y recursos en aquellos lugares que menos recursos alternativos dispongan.

- Asimismo, se apunta el hecho de que cada individuo, según su circunstancia, edad y condición, vive y valora el tiempo y la memoria de una manera diferente. Tal y como Hayden indica en otro de sus trabajos, el 'paisaje urbano como historia pública' (Hayden, 1995) es una herramienta para incidir en el reconocimiento de todos los grupos como sujetos de derecho. Además, el diseñar los distintos espacios asociados a diferentes usos, como espacios equivalentes en dignidad e interés, siempre redundará en la ganancia de autonomía de los grupos de personas reconocidas a la hora de la toma de decisiones.

- Y, finalmente, se recalca que el hecho de que el incorporar la perspectiva de género en la arquitectura y el urbanismo es un indicador más de calidad urbana. Una ciudad o entorno construido que promueve la autonomía de las mujeres que allí viven, mejora todos sus índices de calidad como así lo demuestran las distintas experiencias urbanas entre las que destacan las de Viena, Berlín, Barcelona o Bilbao.

\section{Epílogo}

El trabajo abordado para la incorporación de la perspectiva de género en Castelló no ha podido ser más gratificante e ilusionante. Entendemos que ha coincidido la circunstancia de un equipo técnico municipal excepcional, liderado por Consuelo Leal, ${ }^{17}$ y un equipo de gobierno político respetuoso con las decisiones y recomendaciones técnicas. El equipo asesor en cuestiones de género - redactor de este texto- ha contribuido, fundamentalmente, a que se pongan en marcha los me-

17 El equipo de trabajo y todo el proceso vienen detallado en la memoria previa al Informe de Evaluación de Impacto de Género del PGE de Castelló. Puede consultarse en el siguiente link: https://www.academia.edu/35683992/ INCORPORACI\%C3\%93N_DE_LA_ PERSPECTIVA_DE_G\%C3\%89NERO_ EN_EL_PGE_CASTELL\%C3\%93N_2017_ PERSPECTIVA_DE_G\%C3\%89NERO_ PLAN_GENERAL_ESTRUCTURAL_ CASTELL\%C3\%93N_MEMORIA_INFORME_DE_EVALUACI\%C3\%93N_DE_ IMPACTO_DE_G\%C3\%89NERO [último acceso realizado el 29/03/2018]. canismos de inteligencia colectiva, estableciendo mecanismo de trabajo que permitieran escuchar y hablar, ampliar el marco de trabajo habitualmente empleado e indagar en nuevas posibilidades y cuestiones.

Esta circunstancia ha sido reconocida con el Premio que la Generalitat Valenciana otorga a las actuaciones urbanas y arquitectónicas que han incorporado la perspectiva de género, en la categoría de ciudades de más de 20.000 habitantes, en su segunda edición de 2018, premio que fue entregado por la Honorable Consellera María José Navarro Rubert y recogido por la vicealcaldesa de Castelló, Alicia Brancal y el concejal de urbanismo, Rafael Simó, en acto público el día 5 de marzo de 2018, con gran repercusión en prensa local. 
Todo ello lleva a pensar en el impacto social y político del trabajo profesional y en su incidencia en la vida de las personas. Sería deseable que parte de los hallazgos de este trabajo pudieran ser incorporados en la nueva redacción de la LOTUP —-Ley de Ordenación del Territorio, Urbanismo y Paisaje - en particular la cuestión de los indicadores y de la elaboración de guías.

\section{Agradecimientos}

Queremos hacer explícito nuestro agradecimiento al equipo municipal de redacción del PGE, compuesto por:

- Arquitectos responsables del equipo: Consuelo Leal, Fernando Calduch.

- Arquitectos: José Luis Calabuig, Rosa Pardo, Sara Lloréns, Paula Piñana.

- Técnicos Ambientales: Elena Gil (Ingeniera Agrónoma), Ana Miguel (Ciencias Ambientales).

Y, al equipo de gobierno del Ajuntament de Castelló, en particular a Alicia Brancal y a Rafael Simó. 


\section{Referencias}

Álvarez, E. (2015, 26 de julio). Dolores Hayden 1945. [Entrada de blog] Un día | una arquitecta. Recuperado el 29 de marzo de 2018, de: https:/ / undiaunaarquitecta.wordpress.com/2015/07/26/doloreshayden-1945/.

Arendt, H. (2008). De la historia a la acción. Barcelona: Paidós (Pensamiento contemporáneo, 38).

Ayuntamiento de Castellón de la Plana (2014). Foros de urbanismo del Castellón del siglo XXI: construyendo el futuro. Castellón de la Plana, Castellón: Ayuntamiento de Castellón de la Plana. Recuperado el 24 de agosto de 2018, de: http:/ /www.castello.es/archivos/12/Foros_Urbanismo_p.pdf.

CEPAL - Comisión Económica para América Latina y el Caribe (2014). Informe anual 2013-2014: el enfrentamiento de la violencia contra las mujeres en América Latina y el Caribe. Santiago de Chile: Naciones Unidas. Recuperado el 24 de agosto de 2018, de: https:/ / repositorio.cepal.org/handle/11362/37185.

Col.lectiu Punt 6 (2017). Entorns habitables. Auditoria de seguretat urbana amb perspectiva de gènere a l'habitatge $i$ l'entorn. Barcelona: Diputació de Barcelona. Recuperado el 13 de noviembre de 2017, de: https:/ / issuu.com/punt6/docs/entorns_habitables_cat_final.

Damyanovic, D. (2013). Gender Mainstreaming in Urban Planning and Urban Development. Viena: Urban Development and Planning. Recuperado el 23 de agosto de 2018, de: https:/ /www.wien.gv.at/stadtentwicklung/studien/pdf/b008358.pdf.

Enet, M. (2012). Prologo. En Mansueto, C. y Jaime, E. (2012). Ver para resolver. Transformar las necesidades en proyecto. Buenos Aires: Edición Proyecto Habitar, 4-5.

Ferrero Ibargüen, M.J. (2016, 24 de noviembre). Liza Fior 1962. [Entrada de blog] Un día | una arquitecta 2. Recuperado el 29 de marzo de 2018, de: https://undiaunaarquitecta2.wordpress. com/2016/11/24/liza-fior-1962/.

Freeman, J. (1971). The Women's Liberation Movement: Its origins, structures and ideas. Durham, Carolina del Norte: David M. Rubenstein Rare Book \& Manuscript Library - Duke University (The Atlanta Lesbian Feminist Alliance -ALFA). Recuperado el 24 de agosto de 2018, de: http:/ /library.duke.edu/ digitalcollections/sizes/wlmpc_wlmms01013_wlmms010130010/.

Azara Escrivá, S. y Gil Vila, M.V. (2016). SET para in- troducir la perspectiva de género en el proceso urbano. Valencia: Generalitat Valenciana - Dirección General de Vivienda, Rehabilitación y Regeneración. Recuperado el 24 de agosto de 2018, de: http:/ /www.habitatge.gva.es/documents/20558636/0/SET_cas_ link.pdf.

Hayden, D. (1980). What would a non-sexist city be like? Speculations on housing, urban design, and human work. Signs, 5(3), 170-187.

Hayden, D. (1995). The power of place: urban landscapes as public history. Cambridge, massachussetts: MIT Press.

Mansueto, C. y Jaime, E. (2012). Ver para resolver. Transformar las necesidades en proyecto. Buenos Aires: Edición Proyecto Habitar. Recuperado el 11 de noviembre de 2017, de: https:/ /issuu.com/proyectohabitar/docs/ver_para_resolver.

Leal Jiménez, C. (dir.) (2018). Memoria Justificativa del modelo territorial y urbanístico propuesto. PGE Castelló. Castellón de la Plana: Ajuntament de Castelló. Recuperado el 29 de marzo de 2018, de: https://s3-eu-west-1.amazonaws.com/urbanismo/ MEMORIA+JUSTIFICATIVA.pdf.

Sánchez de Madariaga, I., de Gregorio Hurtado, S. y Novella Abril, I. (s.f.). Perspectiva de Género en las Directrices de Ordenación Territorial del País Vasco. Propuestas de acción. País Vasco: Departamento de Medio Ambiente y Política Territorial - Gobierno Vasco. Recuperado el 24 de agosto de 2018, de: http://www.euskadi.eus/contenidos/informacion/ revision_dot/es_def/adjuntos/Perspectiva de Género en las DOT (ISdM).pdf.

Venturi, R. y Scott Brown, D. (2004). Architecture as signs and systems: for a mannerist time. Cambridge: The Belknap Press of Harvard University Press.

Vives, M. (2014). Diagnóstico, Evaluación y Adecuación del PGOU de Irun en Relación a la Igualdad de Mujeres y Hombres. [Entrada de blog] Projekta Urbes. Recuperado el 11 de noviembre de 2017, de: http:/ / projekta.es/diagnostico-evaluacion-adecuacion-pgou-irun-relacion-mujeres-hombres-igual$\mathrm{dad} /$.

Serrano Lanzarote, B., Mateo Cecilia, C. y Rubio Garrido, A. (eds.) (2017). Género y Política Urbana. Arquitectura y Urbanismo desde la Perspectiva de Género. Valencia: Instituto Valenciano de la Edificación. Recuperado el 23 de agosto de 2018, de: http://www.five.es/descargas/archivos/urbanismo/genero_y_politica_urbana_2017.pdf. 
Álvarez Isidro, E. M. y Gómez Alfonso, C. J. (2018). La incorporación de la perspectiva de género en el Plan General Estructural de Castelló: objetivos, método, acciones y hallazgos. Hábitat y Sociedad, 11, 201-219.

<http://dx.doi.org/10.12795/HabitatySociedad.2018.i11.12> 


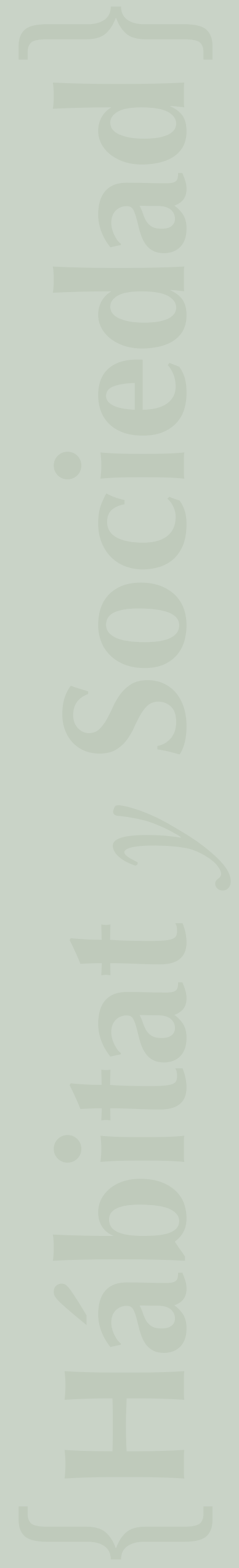

